\title{
Изготовление и исследование изорешеточной гетероструктуры для квантовых каскадных лазеров
}

\author{
(C) В.В. Мамутин ${ }^{1}$, А.П. Васильев ${ }^{1}$, А.В. Лютецкий ${ }^{1}$, Н.Д. Ильинская ${ }^{1}$, Ю.М. Задиранов ${ }^{1}$, \\ А.Н. Софронов ${ }^{2}$, Д.А. Фирсов ${ }^{2}$, Л.Е. Воробьев ${ }^{2}$, Н.А. Малеев ${ }^{1}$, В.М. Устинов ${ }^{1}$ \\ ${ }^{1}$ Физико-технический институт им. А.Ф. Иоффре Российской академии наук, \\ 194021 Санкт-Петербург, Россия \\ ${ }^{2}$ Санкт-Петербургский политехнический университет Петра Великого, \\ 195251 Санкт-Петербург, Россия \\ E-mail: mamutin@mail.ru
}

(Получена 6 декабря 2017 г. Принята к печати 12 декабря 2017 г.)

\begin{abstract}
Описано получение и исследование лазерных характеристик изорешеточной структуры квантового каскадного лазера на подложке фосфида индия, рассчитанного на длину волны $\sim 4.8$ мкм, что соответствует одному из окон прозрачности атмосферы. Гетероструктура была выращена методом молекулярно-пучковой эпитаксии и состояла из тридцати каскадов. Экспериментально получена лазерная генерация при температуpax до $200 \mathrm{~K}$ на длине волны, совпадающей с расчетной, что подтверждает высокое качество интерфейсов и высокую точность исполнения толщин и легирования активной области.
\end{abstract}

DOI: $10.21883 /$ FTP.2018.07.46057.8787

\section{1. Введение}

Квантовые каскадные лазеры (ККЛ) основаны на внутризонных оптических переходах электронов, когда длина волны излучения в них задается не материалом, а зонной структурой сверхрешетки активной области. При этом длина волны излучения может выбираться в широких пределах в зависимости от предполагаемого применения, одно из которых - использование атмосферных окон прозрачности среднего инфракрасного (ИК) диапазона 3-5 мкм для высокоскоростных систем связи, в том числе со спутниками, когда влияние поглощения и турбулентности атмосферы существенно уменьшено по сравнению с видимым и ближним инфракрасным диапазонами. В каскадных лазерах со слоями InGaAs/AlInAs для диапазона длин волн вблизи 5 мкм в основном из-за значений показателя преломления, технологичности и подходящего теплоотвода используются структуры, выращенные на подложках InP [1-5].

Ранее нами ранее было показано, что попадание в требуемую длину волны возможно с использованием дизайна активной области разного типа, например, при использовании напряженно-компенсированных сверхрешеток [1]. В данной работе мы сконцентрировали внимание на решеточно-согласованных гетероструктурах, как наиболее точно контролируемых по составу с помощью методов рентгеновской дифракции и фотолюминесценции. Напряженно-компенсированные гетероструктуры требуют применения неоднозначно интерпретируемых моделей расчета кривых рентгеновской дифракции, в которых изменение толщин в модели приводит к изменению состава и наоборот. В случае изорешеточных сверхрешеток такая неоднозначность исключена ввиду четкого независимого определения состава и толщин толстых слоев тестовых структур, полученных до роста лазерной структуры. Кроме того, этот диапазон технологически удобен в силу состава слоев, позволяющих достигнуть больших толщин эмиттеров без дислокаций и дополнительных напряжений, что бывает необходимо в конкретных структурах каскадных лазеров.

Для оценки лазерной структуры нами использовался упрощенный вариант - так называемые. четырехсколотые образцы, позволяющие определить длину волны и пороги генерации. Для получения лазеров с улучшенными характеристиками ведутся работы по изготовлению оптимальных полосковых структур, которые будут исследованы в дальнейшем.

\section{2. Эксперимент}

\section{1. Выращивание гетероструктур}

Лазерные гетероструктуры были выращены методом молекулярно-пучковой эпитаксии (МПЭ) на установке RIBER Compact-21 TM. Ростовые температуры активных областей лазерных структур составляли $500^{\circ} \mathrm{C}$, и при росте использовались слабо Аs-стабилизированные условия. Лазерная структура была разработана с решеточно-согласованной активной областью и эмиттерами с учетом данных из работ $[2,3]$ и наносилась на подложку $n-\operatorname{InP}(100): \mathrm{Sn}$, легированную до $2 \cdot 10^{17} \mathrm{~cm}^{-3}$.

Активная область квантового каскадного лазера состояла из 30 периодов. Один период содержал последовательность из 18 чередующихся слоев AlInAs/GaInAs, (в нанометрах):

$$
\begin{aligned}
& 3.0 / \underline{4.0} / 2.2 / \underline{4.7} / 1.5 / \underline{0.9} / 5.0 / \underline{1.9} / 2.8 / \underline{1.9} / 2.3 / \\
& / \underline{2.0} / \mathbf{2 . 0} / \underline{\mathbf{2 . 0}} / \mathbf{2 . 2} / \underline{\mathbf{2 . 2}} / 2.3 / \underline{2.3},
\end{aligned}
$$

где подчеркнуты значения толщин слоев квантовых ям GaInAs и выделены жирным значения толщин легированных слоев. Эти слои были легированы кремнием с концентрацией $n=4 \cdot 10^{17} \mathrm{~cm}^{-3}$. 
Вся структура (указана последовательность от подложки по направлению роста, в скобках приводится уровень легирования) состояла из следующих слоев: $300 \mathrm{Hм} \mathrm{-} \mathrm{волноводный} \mathrm{слой} \mathrm{Ga}_{0.47} \mathrm{In}_{0.53} \mathrm{As}$ $\left(1 \cdot 10^{17} \mathrm{~cm}^{-3}\right), \quad 1356 \mathrm{Hм}-(30$ периодов $)$ активная зона, 200 нм - волноводный слой $\mathrm{Ga}_{0.47} \mathrm{In}_{0.53} \mathrm{As}$ $\left(1 \cdot 10^{17} \mathrm{~cm}^{-3}\right)$, затем начинается толстый эмиттер: $600 \mathrm{HM}-\mathrm{Al}_{0.48} \mathrm{In}_{0.52} \mathrm{As} \quad\left(2 \cdot 10^{17} \mathrm{~cm}^{-3}\right), \quad 700 \mathrm{HM}-$ $\mathrm{Al}_{0.48} \mathrm{In}_{0.52} \mathrm{As}\left(3 \cdot 10^{17} \mathrm{~cm}^{-3}\right), 1200 \mathrm{HM}-\mathrm{Al}_{0.48} \mathrm{In}_{0.52} \mathrm{As}$ $\left(7 \cdot 10^{18} \mathrm{~cm}^{-3}\right)$, и верхний ,подконтактный“ сильно легированный слой 50 нм Ga $\mathrm{Ga}_{0.47} \mathrm{In}_{0.53} \mathrm{As}\left(2 \cdot 10^{19} \mathrm{~cm}^{-3}\right)$.

При выборе количества периодов активной области необходимо было учитывать зависимость пороговой плотности тока от потерь, усиления и фактора ограничения:

$$
J_{\mathrm{thr}}\left(N_{p}=\left(\alpha_{m}+\alpha_{w}\right) /\left(g \cdot \Gamma\left(N_{p}\right)\right),\right.
$$

где $J_{\mathrm{thr}}\left(N_{p}\right)$ - пороговая плотность тока, $\alpha_{m}$ - потери на отражение $\left(\alpha_{m}=1 / L \cdot(\ln \mathrm{R})\right.$, где $L-$ длина резонатора, $R$ - коэффициент отражения зеркал), $\alpha_{w}-$ потери в волноводе, $g \cdot J_{\mathrm{thr}}-$ коэффициент усиления, $\Gamma\left(N_{p}\right)$ - коэффициент оптического ограничения, $N_{p}-$ число периодов активной области. Оказалось, что лазеры с $N_{p}$ меньше 6 не дают генерации при комнатной температуре, при увеличении периодов с 12 до 75 пороговая плотность тока при $300 \mathrm{~K}$ уменьшается с 16 до $8 \mathrm{\kappa} \mathrm{A} / \mathrm{cm}^{2}$ соответственно, а теоретические предсказания хорошо согласуются с экспериментом и дают $J_{\mathrm{thr}} \leq 10 \mathrm{\kappa A} / \mathrm{cm}^{2}$ при числе периодов бо́льшем 20 [6]. Кроме того, зависимость пороговой плотности тока от температуры и числа периодов перестает меняться для $N_{p} \geq 20$ (характеристическая температура $T_{0} \geq 100 \mathrm{~K}$ ). Квантовые каскадные лазеры ведут себя с температурой как и обычные полупроводниковые лазеры с пороговой плотностью тока

$$
J_{\mathrm{thr}}(T)=J_{0} \exp \left(T / T_{0}\right),
$$

со значениями $T_{0}$ выше $100 \mathrm{~K}$ для оптимизированных конструкций, что является очень большим значением для других полупроводниковых лазеров с обычным значением $T_{0}$ ниже $100 \mathrm{~K}$.

Кроме того, для $N_{p} \geq 12$ дифференциальная квантовая эффективность становится больше единицы, а рабочее напряжение при этом не становится „слишком“ большим (3-10 В для 10-40 периодов) [6]. Таким образом, оптимальное количество периодов лежит в пределах 20-40 для удовлетворения всех предполагаемых требований. Техническая задача роста соответствующего количества слоев $(\sim 600)$ не представляет трудности для современной установки МПЭ. Но с точки зрения времени роста, расхода материалов и износа установки требуется уменьшать по возможности количество слоев, в результате мы приходим к минимально необходимому количеству $\sim 30$. Такое число периодов и было выбрано для наших структур. Отметим, что использование большего количества периодов (60), как доказано в работе [7], „не приводит к желаемому увеличению квантовой эффективности и оптической мощности, а лишь приводит к увеличению электрического напряжения и дополнительному разогреву лазера. Периферийные каскады фактически не работают".

Из выращенной структуры были изготовлены тестовые четырехсколотые образцы с размерами от $\sim 400 \times 400$ мкм до $\sim 550 \times 550$ мкм.

\section{2. Измерение спектров спонтанного и стимулированного излучения}

Измерения спектров излучения тестовых образцов производилось на установке на базе фурье-спектрометра Bruker Vertex 80v. Образцы монтировались в азотный криостат с выходным оптическим окном из $\mathrm{ZnSe}$ и устанавливались в фокусе параболического зеркала входного порта спектрометра. Излучение, проходя через спектрометр, в котором использовался светоделитель из $\mathrm{KBr}$, собиралось на входном окне охлаждаемого жидким азотом фотоприемника на основе кадмий-ртуть-теллура (КРТ).

Токовая накачка образцов осуществлялась с помощью драйвера на электронной ключевой схеме с быстродействующим мощным полевым транзистором в импульсном режиме с длительностью импульса от 200 нс до нескольких микросекунд и частотой до 10 кГц. Измерения спектров спонтанного излучения производились в режиме пошагового сканирования спектрометра с многократным усреднением сигнала фотоприемника, измеряемого импульсным синхронным детектором SR250 в течение действия импульса тока, и дальнейшей оцифровкой АЦП спектрометра. Спектральное разрешение составляло $8 \mathrm{~cm}^{-1}$.

Спектры стимулированного излучения измерялись в режиме пошагового сканирования с внешней синхронизацией по токовому импульсу, при этом сигнал фотоприемника оцифровывался без предобработки непосредственно АЦП спектрометра, позволяя восстанавливать разрешенные по спектру временны́е зависимости интенсивности излучения в течение действия импульса тока. Спектральное разрешение составляло $0.3 \mathrm{~cm}^{-1}$.

Измерения амплитуды импульсов тока и падения напряжения на структуре производились с помощью цифрового осциллографа (ток измерялся по падению напряжения на малом добавочном сопротивлении). Интегральная интенсивность спонтанного излучения измерялась импульсным синхронным детектором, интенсивность стимулированного излучения - цифровым осциллографом.

\section{3. Эксперименальные результаты и их обсуждение}

На всех тестовых образцах была получена лазерная генерация. На рис. 1 представлены типичные вольт-амперные характеристики лазерной структуры вместе с зависимостями интенсивности излучения от тока накачки для трех температур теплоотвода. Вольт-амперные 


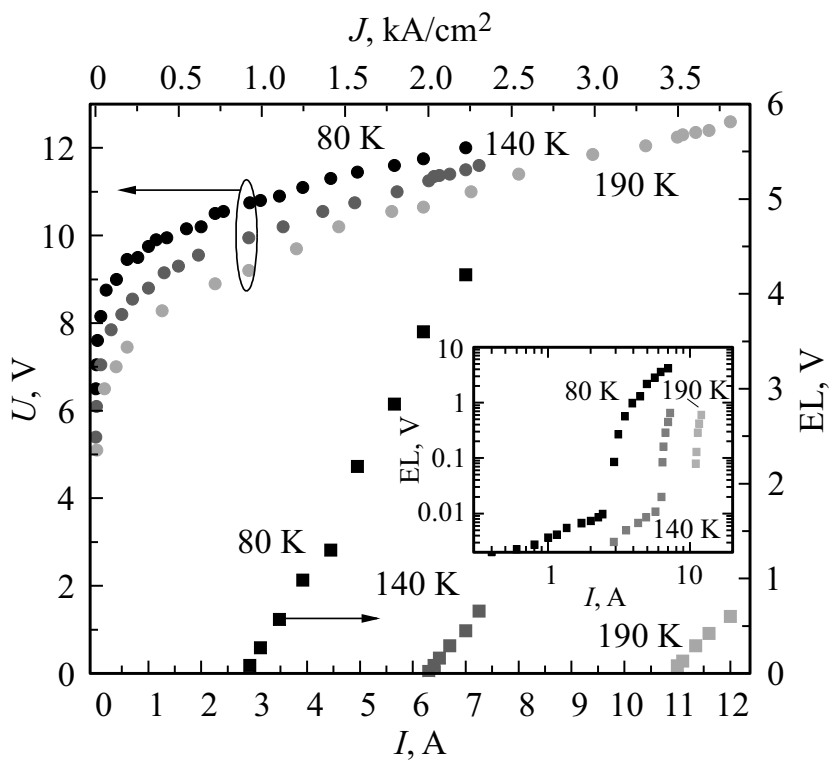

Рис. 1. Вольт-амперные характеристики и токовые зависимости интегральной интенсивности излучения для одного из тестовых образцов при температурах теплоотвода 80, 140 и $190 \mathrm{~K}$. На вставке показаны сигналы фотоответа в двойном логарифмическом масштабе при тех же температурах.

характеристики являются характерными для вертикального транспорта в каскадных структурах и показывают общее падение напряжения на структуре в рабочем режиме, не превышающее $12 \mathrm{~B}$.

В допороговом режиме была зафиксирована спонтанная люминесценция структуры. Лазерная генерация возникала при достижении порогового тока через структуру и характеризовалась резким ростом интегральной интенсивности детектируемого сигнала фотоответа (см. вставку на рис. 1). Спектры спонтанной люминесценции, измеренные при трех значениях температуры теплоотвода, приведены на рис. 2. Полная ширина на половине высоты $F W H M$ основного максимума спектра спонтанной эмиссии при $T=80 \mathrm{~K}$ составляет 10 мэВ. С ростом температуры $F W H M$ максимум имеет небольшую тенденцию к увеличению (см. вставку на рис. 2), а сам максимум немного смещается в длинноволновую сторону, что говорит о вертикальном (недиагональном) характере переходов в активной зоне [8,9]. Температурный сдвиг и уширение спектра спонтанной люминесценции объясняются термическим уширением распределения неравновесных носителей в активной области, что может приводить к проявлению эффектов непараболичности закона дисперсии и увеличению вероятности рассеяния электронов, в число механизмов которого входит также и рассеяние на интерфейсах. Полученные значения $F W H M$ линии спонтанной люминесценции являются характерными для качественных квантово-каскадных лазеров $[6,8,9]$, что говорит о высоком совершенстве гетерограниц и низком уровне фонового легирования активной области выращенной структуры.
Типичные спектры лазерной генерации, измеренные при $T=80 \mathrm{~K}$ для двух значений тока через структуру, показаны на рис. 3. В исследованных тестовых четырехсколотых образцах рабочими оптическими модами являются моды полного внутреннего отражения. Измерения время-разрешенных спектров показывают наличие „Перескоков“ между конкурирующими модами или генерацию сразу на нескольких модах в разные моменты времени в течение действия импульса тока (на рис. 3 показаны наиболее репрезентативные срезы). Из-

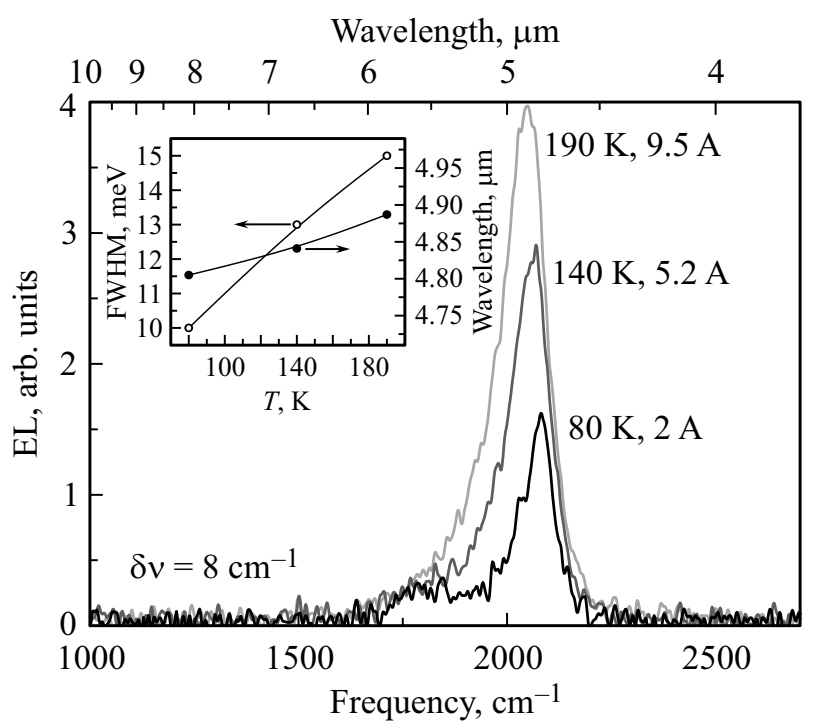

Рис. 2. Спектры спонтанной люминесценции, измеренные при температурах теплоотвода 80,140 и $190 \mathrm{~K}$ в допороговом режиме (значения тока подписаны у соответствующих кривых). На вставке - зависимости $F W H M$ (левая ось) и положения спектрального максимума (правая ось) спонтанной люминесценции от температуры.

Wavelength, $\mu \mathrm{m}$

$\begin{array}{llllllllll}4.90 & 4.89 & 4.88 & 4.87 & 4.86 & 4.85 & 4.84 & 4.83 & 4.82 & 4.81\end{array}$

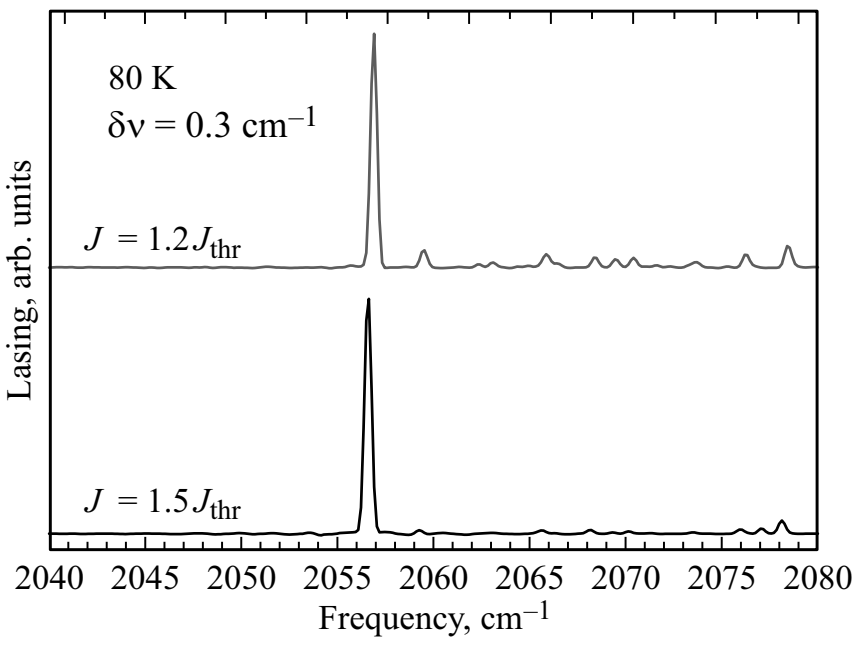

Pис. 3. Типичные спектры лазерной генерации, измеренные при температуре теплоотвода $80 \mathrm{~K}$ при токах, превышающих пороговый на 20 и 50\% (каждая кривая нормирована на свой максимум). 


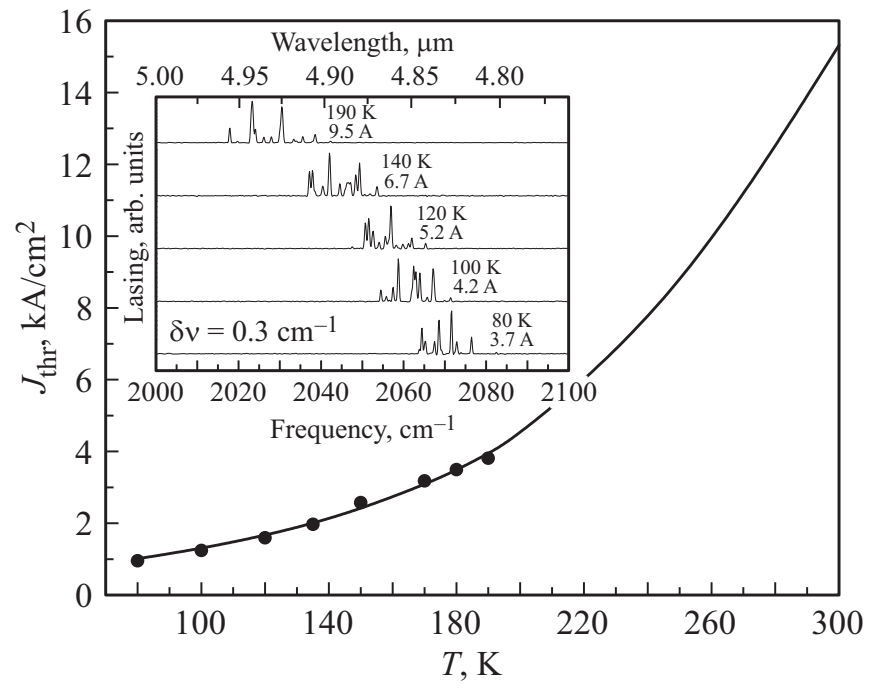

Рис. 4. Температурная зависимость порогового тока (точки эксперимент, линия соответствует экспоненциальному росту по закону $\sim \exp \left(T / T_{0}\right)$ с $\left.T_{0} \approx 81 \mathrm{~K}\right)$. На вставке показано смещение спектров генерации с температурой (значения температур и токов подписаны у кривых).

меренная $F W H M$ линии лазерной генерации ограничена спектральным разрешением спектрометра.

Измеренная зависимость плотности порогового тока от температуры (рис. 4) хорошо описывается выражением (1) с характерной плотностью тока при нулевой температуре $J_{0} \approx 0.59$ кА/ $\mathrm{cm}^{2}$ и характерной температурой $T_{0} \approx 81 \mathrm{~K}$. На вставке рис. 4 показан температурный сдвиг спектров генерации. При нагреве от 80 до $190 \mathrm{~K}$ основной максимум спектра лазерной генерации сдвигается от 4.82 до 4.95 мкм, а пороговый ток возрастает от $\sim 0.95$ до $\sim 3.5 \mathrm{\kappa A} / \mathrm{cm}^{2}$.

Лазерная генерация была экспериментально зафиксирована вплоть до $200 \mathrm{~K}$. Дальнейшее увеличение тока накачки для исследованных тестовых образцов было нецелесообразно, так как могло привести к выходу из области предельно допустимых значений пиковой (и средней при больших частотах) мощности как для самого образца, так и для драйвера.

\section{4. Заключение}

Исследованы лазерные характеристики выращенной с помощью молекулярно-пучковой эпитаксии изорешеточной структуры квантового каскадного лазера на подложках фосфида индия, рассчитанного на длину волны $\sim 4.8$ мкм, соответствующей окну прозрачности атмосферы. В эксперименте получена лазерная генерация при температурах вплоть до $200 \mathrm{~K}$ на длине волны, совпадающей с расчетной, что подтверждает высокое качество интерфейсов и прецизионную точность толщин и легирования активной области ККЛ. Исследованы спектральные характеристики спонтанного и стимулированного излучения. Полуширина пика спонтанно- го излучения соответствует высококачественным ККЛ. Плотность порогового тока при температуре жидкого азота для исследованных тестовых образцов составила $\sim 0.95 \kappa \mathrm{A} / \mathrm{cm}^{2}$.

Работа выполнена при частичной финансовой поддержке Министерства образования и науки России (государственное задание № 3.933.2017/4.6 и № 3.6153.2017/7.8)

\section{Список литературы}

[1] В.В. Мамутин, В.М. Устинов, J. Boetthcher, H.Kuenzel. ФТП, 44, 995 (2010).

[2] J. Faist, A. Tredicucci, F. Capasso, C. Sirtori, D.L. Sivco, J.N. Baillargeon, A.L. Hutchinson, A.Y. Cho. IEEE J. Quant. Electron., 34, 336 (1998).

[3] V.V. Mamutin, V.M. Ustinov, N.D. Ilyinskaya, M.V. Baydakova, B.Ya. Ber, D.Yu. Kasantsev. J. Phys.: Conf. Ser., 291, 012008 (2011).

[4] A. Tsekoun, R. Go, M. Pushkarsky, M. Razeghi, C.K.N. Patel. PNAS (Proc. Natl. Acad. Sci. USA), 103, 4831 (2006).

[5] M. Razeghi, S. Slivken, Y. Bai, B. Gokden, S.R. Darvish. New J. Phys., 11, 125017 (2009).

[6] C. Gmachl, F. Capasso, D.L. Sivco, A.Y. Cho. Rep. Prog. Phys., 64, 1533 (2001).

[7] А.В. Бабичев, Н.А. Пихтин, И.С. Тарасов, Е.В. Никитина, А.Н. Софронов, Д.А. Фирсов, Л.Е. Воробьев, И.И. Новиков, Л.Я. Карачинский, А.Ю. Егоров. ФТП, 50, 1320 (2016).

[8] J. Faist, F. Capasso, D.L. Sivco, C. Sirtori, A.L. Hutchinson, A.Y. Cho. Appl. Phys. Lett., 67, 3057 (1995).

[9] J. Faist, F. Capasso, S. Sirtori, D.L. Sivco, J.N. Baillargeon, A.L. Hutchinson, S.-N.G. Chu, A.Y. Cho. Appl. Phys. Lett., 68, 3680 (1996).

Редактор А.Н. Смирнов

\section{Lattice matched structure growth and investigation for quantum cascade lasers}

V.V. Mamutin ${ }^{1}$, A.P. Vasilyev ${ }^{1}$, A.V. Lyutetskiy ${ }^{1}$, N.D. Ilyinskaya ${ }^{1}$, Yu.M. Zadiranov ${ }^{1}$, A.N. Sofronov' ${ }^{2}$, D.A. Firsov' ${ }^{2}$, L.E. Vorobjev ${ }^{2}$, N.A. Maleev' ${ }^{1}$, V.M. Ustinov ${ }^{1}$

${ }^{1}$ loffe Institute, 194021 St. Petersburg, Russia

${ }^{2}$ Peter the Great St. Petersburg Polytechnic University, 195251 St. Petersburg, Russia

Abstract We have demonstrated MBE growth and investigation of lattice matched quantum cascade laser structure on the indium phosphide substrate with 30 cascades designed for $\sim 4.8 \mu \mathrm{m}$ wavelength for the atmospheric window in this spectral range. The lasing was experimentally observed at the temperatures up to $200 \mathrm{~K}$ and have demonstrated exactly the expected spectral line position. This confirms high heterointerface quality and high precision control of the layer thicknesses and doping levels in the laser active region. 